\title{
Inflammatory bowel disease reveals the kinase activity of KSR1
}

\author{
Richard Kolesnick and H. Rosie Xing
}

Laboratory of Signal Transduction, The Sloan-Kettering Institute, Memorial Sloan-Kettering Cancer Center, New York, New York, USA.

\begin{abstract}
Kinase suppressor of Ras-1 (KSR1) is a recently identified member of the EGFR-Ras-Raf-1-MAPK signaling pathway. A new study demonstrates that KSR1 protects intestinal epithelium from TNF- $\alpha$-induced apoptosis, abrogating inflammatory bowel disease (IBD) (see the related article beginning on page 1272). Since its discovery, there has been disagreement as to whether KSR1 possesses intrinsic kinase activity. Using transgenic mouse models and genetically modified mouse colon epithelial cells, Polk and coworkers show that the kinase activity of KSR1 is off in normal colon epithelial cells, becoming activated only at the onset of IBD. They also provide strong evidence that KSR1 kinase activity is essential for anti-apoptotic protection of the intestinal epithelium. These new data in support of KSR1 as a kinase highlight an ongoing debate as to whether KSR1 does indeed serve as a specific kinase in transphosphorylating and transactivating c-Raf-1 toward MEK1.
\end{abstract}

TNF- $\alpha$ appears to play a key role in the pathogenesis of inflammatory bowel disease (IBD), regulating the balance between anti- and pro-apoptotic pathways in intestinal epithelial cells (1). TNF- $\alpha$ overexpression has been found sufficient for IBD induction in a mouse model, (2) and anti-TNF- $\alpha$ therapies have been shown to reverse IBD in patients (3). Thus, understanding TNF- $\alpha$ signaling in intestinal epithelial cells is crucial for the development of mechanism-based therapies for IBD and other TNF- $\alpha$-mediated intestinal disorders. In this issue of the JCI, Yan, Polk, and colleagues (4) demonstrate that kinase suppressor of Ras-1 (KSR1) functions as a molecular switch, providing an essential survival signal that protects intestinal epithelial cells from TNF- $\alpha$-induced apoptosis, and consequently, IBD.

Regulation of TNF- $\alpha$-mediated cell survival and proliferation versus apoptosis is not well understood. In epithelial cells, transient Ras/c-Raf-1-dependent activation of the MAPK cascade appears to mediate anti-apoptotic function upon

Nonstandard abbreviations used: IBD, inflammatory bowel disease; IMP, impedes mitogenic signal propagation; Ki-KSR1 ${ }^{\text {D683/D700 }}$, kinase-inactive KSR1; KSR1, kinase suppressor of Ras-1; MCE, mouse colon epithelial; PP2A, protein phosphatase 2A, YAMC, young adult mouse colon.

Conflict of interest: The authors have declared that no conflict of interest exists.

Citation for this article: J. Clin. Invest. 114:1233-1237 (2004). doi:10.1172/JCI200423441.
TNF- $\alpha /$ TNF receptor 1 interaction (5). Prior studies by Yan et al. $(6,7)$ defined KSR1 as a pivotal transducer of TNF- $\alpha-$ stimulated c-Raf- 1 activation of ERK1/2 MAPKs and NF-KB in young adult mouse colon (YAMC) epithelial cells. These data also indicated that KSR1 kinase activity was required for c-Raf-1 phosphorylation and activation $(6,7)$. The current studies (4) extend these observations to in vivo models using genetically modified mice and primary cultures of colon epithelium derived thereof. Strong evidence indicates that KSR1, via c-Raf-1, confers antiapoptotic protection to epithelium within the murine colon and that KSR1 kinase activity is key to this action.

\section{Evidence that KSR1 kinase activity transactivates c-Raf-1}

KSR1 was originally identified as a ceramide-activated protein kinase (8) prior to its being defined in Drosophila melanogaster and Caenorbabditis elegans as a positive modulator of Ras-MAPK signaling either upstream of or parallel to c-Raf- 1 $(9-11)$. There is a general agreement that KSR1 functions to coordinate signaling of the Ras GTPase through to its downstream effector, c-Raf-1. KSR1 interacts with several proteins that possess kinase activity, including c-Raf-1, MEK1, MAPK, C-TAK1 (12-17) and with protein phosphatase 2A (PP2A) (18). In addition, KSR1 associates with 14-3-3 and heat shock proteins (14, $17,19)$, with the $\beta \gamma$ subunits of $G$ proteins
(20), and with IMP (impedes mitogenic signal propagation) (21). A recent set of elegant studies showed that in quiescent cells, C-TAK1 constitutively binds to and phosphorylates KSR1 on Ser392, conferring 14-3-3 association, resulting in the retention of KSR1 and MEK1 in the cytosol (17) (Figure 1A). Upon stimulation with growth factors, Ser392 is dephosphorylated by PP2A, leading to partial release of KSR1 from 14-3-3 and rapid KSR1 translocation to the membrane (18), where KSR1-associated MEK1 and MAPK are presented to $\mathrm{c}$-Raf- 1 bound to Ras GTP. This event is negatively regulated by IMP which uncouples signal transduction from c-Raf-1 to MEK1 by inactivating KSR1 (21) (Figure 1B). While these studies lead to the hypothesis that KSR1 serves as a molecular scaffold to localize all constituents of the MAPK signaling cascade at the membrane, presumably thereby assembling the active signaling complex, this paradigm provides no mechanistic insight into the manner by which c-Raf- 1 becomes active toward its target, MEK1.

The primary amino acid sequence predicts KSR1 orthologs from all species represent protein kinases. The C-termini of KSR1 proteins contain the 11 conserved kinase subdomains found in all known protein kinases (9). The presence of an HKDLR motif in subdomain VI of the Drosophila and C. elegans KSR1 orthologs suggests that they represent tyrosine kinases $(10,11)$, while mammalian KSR1 possesses an HKDLK motif typical of serine/threonine kinases (9). Although the ATP-binding domain of C. elegans and Drosophila KSR1 is conserved, the Lys residue normally involved in ATP binding in almost all kinases is substituted in mammalian KSR1 homologs with Arg (discussed below).

Four research teams have reported specific kinase activity toward c-Raf- 1 associated with KSR1 isolated from diverse cell types, including COS-7 kidney fibroblasts, A431 cervical carcinoma, HL-60 promyelocytic leukemia, and C16 glioma as well as 
A

PM

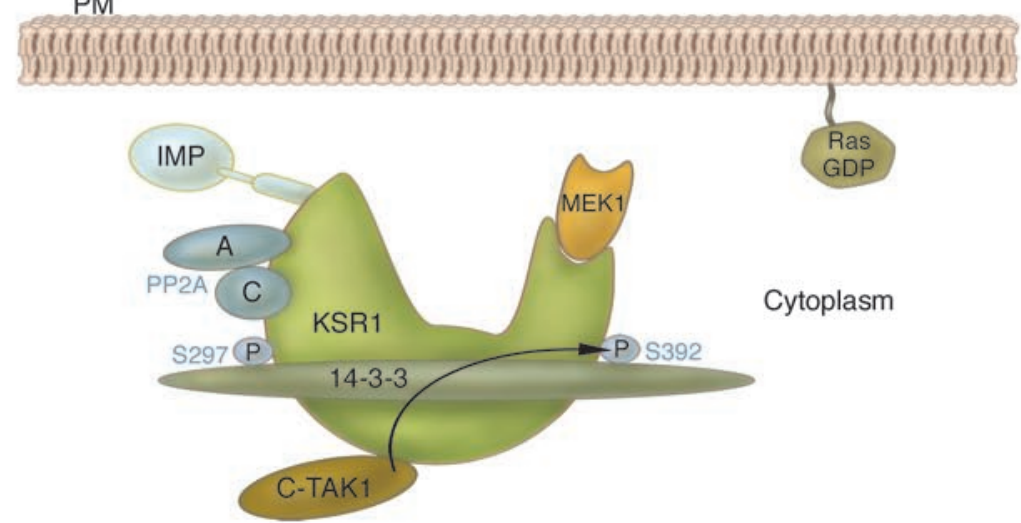

B
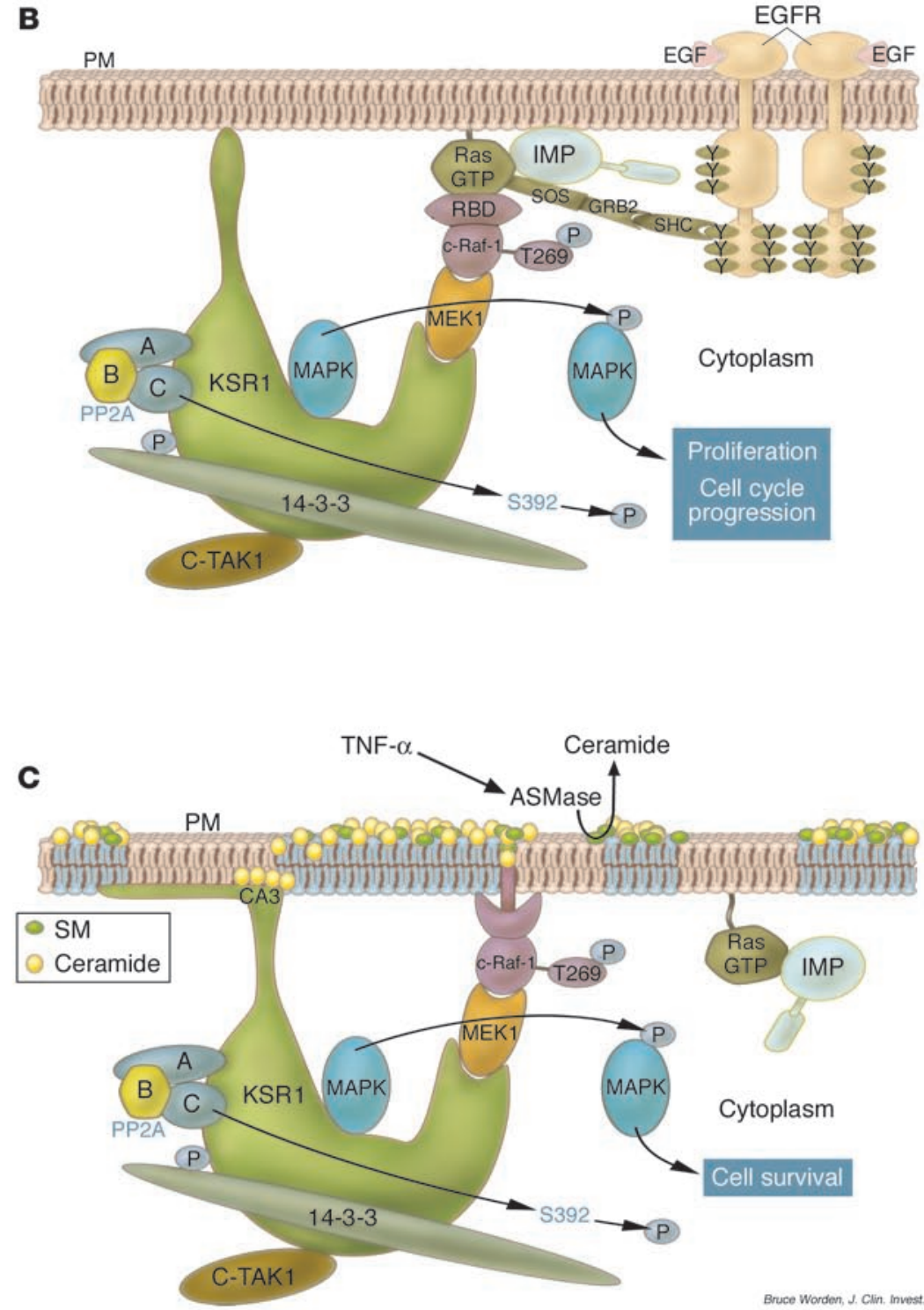

Figure 1

Model for the regulation of C-Raf-1 signaling by KSR1. (A) In quiescent cells, KSR1 is phosphorylated on Ser297 by an unknown kinase and on Ser392 by C-TAK1, creating docking sites for 14-3-3. The 14-3-3-KSR1 interaction results in sequestration of KSR1 and MEK1, to which it is constitutively bound, in the cytosol (17). Both KSR1 and its target, c-Raf-1, are also constitutively bound to the PP2A core enzyme (subunit A and catalytic subunit C) (18). In addition, IMP interacts with the $\mathrm{N}$-terminus of KSR1, maintaining KSR1 in an inactive state (21). (B) During growth factor stimulation (such as via EGF), KSR1 and c-Raf-1 acquire the PP2A regulatory $B$ subunit, and the holoenzyme dephosphorylates the Ser392 site of KSR1 and the Ser259 site of c-Raf-1, leading to partial release of 14-3-3 from each protein (18). In the case of KSR1, this opens up the MAPK-binding site, while for c-Raf-1, it confers binding to activated Ras at the plasma membrane. In addition, Ras GTP binds IMP, relieving the inhibition of KSR1 (21). Dissociation of KSR1 from IMP and displacement of 14-3-3 on Ser392 leads to rapid translocation of KSR1 to the plasma membrane (PM) and localizes MEK1 and MAPK to membrane-bound c-Raf-1. There is debate as to the mechanism of c-Raf- 1 activation through KSR1, whether activation occurs via a scaffold or kinase function. The scaffold model argues that KSR1-bound c-Raf-1 becomes activated by an unknown mechanism $(15,19,41)$ while the KSR1 kinase model ascribes KSR1-mediated transphosphorylation at Thr269 as the mechanism of c-Raf-1 kinase activation (8, 23). Active c-Raf-1 stimulates MEK1, which in turn activates MAPK, conferring cell proliferation. RBD, Ras-binding domain; Y on EGFR, autophosphorylated tyrosine residues; SOS, son of sevenless; SHC, src homologous and collagen protein. (C) At the onset of IBD, KSR 1 is activated and confers colon epithelial cell survival. In colon epithelial cells, ligation of TNF receptor 1 by TNF- $\alpha$ leads to acid sphingomyelinase (ASMase) activation, ceramide generation, and KSR1 and c-Raf-1 translocation to the PM, perhaps by binding of their $\mathrm{C} 1 \mathrm{~b}$ domains to ceramide. There is emerging evidence in other cell types that ceramide converts sphingolipid-enriched microdomains (rafts) into large platforms into which signaling proteins compartmentalize $(28,29)$. However, it is not known if KSR1 translocates into a ceramide-rich platform. In the current studies, Polk and coworkers (4) provide strong evidence that in IBD the ceramide-KSR1 interaction triggers KSR1 kinase activity, resulting in transphosphorylation and transactivation of c-Raf-1, which in turn activates MEK1 and MAPK, conferring epithelial cell survival. SM, sphingomyelin; CA3, the $\mathrm{C} 1 \mathrm{~b}$ homologous domain of KSR1. 
YAMC and mouse colon epithelial (MCE) cell lines $(6,7,22-27)$. The kinase activity was shown to be initiated by two distinct mechanisms, through growth factor receptor tyrosine kinases, such as EGFR (23, $26)$, or by cytokines such as TNF- $\alpha$, likely via direct interaction of KSR1 with the second messenger ceramide $(4,6,7)$ (Figure $1 \mathrm{C}$ ). The latter mode of activation was reported to be mediated via the KSR1 C1b lipid-binding domain (28-30). The kinase activity associated with KSR1 appears to be of the proline-directed class of serine/threonine protein kinases that phosphorylate Thr, which is immediately followed by a Pro residue $(8,31)$. The target recognition site was originally defined, using a series of peptides based on the amino acid sequence surrounding the Thr669 site of the EGFR, to be an unusual proline-directed variant, as there was preference for a TLP motif with a Lys interposed between the target Thr and Pro residues (31). Subsequently, direct sequencing revealed that a ceramide-activated kinase activity renatured from HL-60 cells and bovine brain phosphorylated recombinant c-Raf-1 on Thr269, which is contained within a TLP motif (Figure 1B) $(8,31-34)$. Once recombinant KSR1 became available, immunopurified KSR1 from TNF- $\alpha$-, ceramide- or EGF-stimulated cells similarly phosphorylated recombinant c-Raf- 1 isolated from c-src and V12 Ras-overexpressing Sf9 cells or purified to apparent homogeneity from COS-7 cells on this site $(4,6-8,23,26)$. The suggestion that KSR1 activity was transphosphorylating rather than enhancing c-Raf-1 intrinsic autokinase activity was confirmed by transphosphorylation of a kinase-inactive c-Raf-1 substrate (c-Raf-1-K375M) (23). Not only can immunopurified KSR1 phosphorylate c-Raf- 1 in vitro, but it also transactivates c-Raf-1 toward MEK1, as measured by direct MEK1 phosphorylation and MEKinduced MAPK activation (4, 6, 7, 23-26). This activity does not appear to be a contaminant of immunopurified KSR1 since multiple high salt washes, which result in a KSR1 preparation devoid of background silver-stained proteins or ${ }^{35} \mathrm{~S}$-labeled proteins isolated from cells preincubated with ${ }^{35} \mathrm{~S}$-methionine, do not alter specific activity toward c-Raf-1 $(23,26)$. Moreover, mutation of two conserved Asp residues (D683 and D700) required for ATP catalysis and phosphotransfer to Ala, a classic approach to generate a dead kinase, abolished both the transphosphorylating and transactivating activity of KSR1 isolated from mul- tiple cell lines $(4,6-8,22-24,26)$. In fact, this "kinase-dead" KSR1 mutant serves a dominant-negative function. Numerous different biologic readouts have now been assessed comparing wild-type KSR1 with this dominant-negative KSR1 in mammalian systems. In general, wild-type KSR1 overexpression increases signaling through the MAPK cascade and MAPK-mediated biologic responses, while dominant-negative kinase-dead KSR1 abrogates MAPK signaling and biologic readouts, including BCL2-antagonist of cell death-mediated (BAD-mediated) apoptosis in COS-7 cells (35), vitamin D-mediated differentiation of HL-60 cells (22), survival of YAMC cells upon TNF- $\alpha$ treatment $(6,7)$, and EGFRdriven A431 tumorigenesis in vitro and as xenografts in nude mice (36).

\section{Revisiting the evidence against the KSR1 kinase model}

While these observations, generated collectively by several groups, support the contention that KSR1-associated kinase activity detected using in vitro kinase assays is likely inherent to this protein, a number of groups have been unable to detect a kinase activity associated with KSR1 and thus conclude that KSR1 acts exclusively as a scaffold. The reasons for these conflicting observations are not apparent from the available data, although differences in protocols and systems utilized may have contributed to this experimental outcome. The work of Yan et al. (4) presented in this issue reveals the kinase activity of KSR1 to be off in normal colon epithelial cells, and that it only becomes activated at the onset of IBD. Similarly, KSR1 activity is undetectable prior to EGF stimulation of COS-7 cells, or ceramide or TNF- $\alpha$ stimulation of YAMC cells $(7,26)$. Further, the kinase activity of KSR1 is detected only transiently $(4,6,7,23,25,26)$. For instance, during EGF stimulation of COS-7 or A431 cells, KSR1 activity peaks at 3 minutes and returns to baseline by 10 minutes (26). Hence, it would be easy to miss the activity unless the activation conditions used were defined in great detail.

Another experimental procedure that may have contributed to the failure of some groups to detect KSR1 kinase activity is the use of overexpression systems that far exceed physiological levels. Several studies show that, under conditions of marked overexpression, both biological activity and cellular localization of KSR1 are altered, often yielding effects that are the opposite of low-dose expression (37-39). In contrast, the groups that claim to detect KSR1 activity demonstrated such activity in immunoprecipitates of endogenous KSR1 from MCE, YAMC, A431, and HL-60 cells $(6,7,22,24,26)$. Additionally, the kinase function of KSR1 is cell type specific and context dependent. For instance, while EGF-stimulated MAPK activation in COS-7 cells, A431 cells, and mouse embryonic fibroblasts required KSR1 $(26,40)$, MAPK activation by EGF in YAMC and MCE cells was KSR1-independent. Nonetheless, KSR1 kinase activity was essential for TNF- $\alpha-$ and ceramide-induced MAPK activation in these latter lines $(6,7)$.

Of the five groups that have failed to detect KSR1-associated kinase activity, only two groups conducted studies in stimulated cells $(13,15,17,19,41,42)$. In one instance, the inability to detect ceramide activation of KSR1 was only briefly mentioned in the discussion as "data not shown," and hence an analysis of that experimental protocol is not possible (13). However, Morrison and coworkers reported a thorough set of studies that examined the above parameters in detail and yet failed to detect ceramide activation of KSR1 kinase (41). While the reasons for this finding are not apparent, these studies also failed to detect direct binding of the KSR1 C1b domain (CA3 domain) to ceramide (43). The latter observation is incompatible with recent reports on the function of the C1b domain of KSR1. Gulbins and colleagues showed that recombinant KSR1 C1b domain binds long-chain natural ceramide with high affinity, but not other lipids, including dihydroceramide, arachidonic acid or sphingomyelin (28). These observations were confirmed by Sobota and coworkers (29). Further, the KSR1 C1b domain has been employed as a probe to localize ceramide-enriched membrane rafts on the surface of CD95-stimulated Jurkat (28) and Fc $\gamma$ RII cross-linked U937 cells (29). Moreover, neutralization of surface ceramide by preincubation with a KSR1 C1b-domain polypeptide abolished ceramide-mediated clustering of CD95 and apoptosis in human Jurkat cells and JY B lymphocytes (28), and in vivo prevented ceramide-mediated CD95 clustering, apoptosis of hepatocytes, and death of mice after intravenous anti-CD95 antibody (28). These observations strongly support direct association with the $\mathrm{C} 1 \mathrm{~b}$ domain as key to the biologic impact of ceramide via KSR1.

Lastly, the systems used to assess KSR1 activity differ greatly. The strongest evi- 
dence against there being a kinase activity associated with KSR1 is derived from nonmammalian genetic model systems (Drosophila, C. elegans, and Xenopus). Particularly compelling are the studies of Guan and coworkers displaying reconstitution of the activated Ras phenotype by overexpressed kinase-dead C. elegans ksr-1 generated by mutating either the putative $\mathrm{Mg}^{2+}$-ATP-binding site (K503M) or the catalytic nucleophile Asp residue (D618A) (19). However, in these studies, neither copy number nor expression levels of wildtype or kinase-dead ksr-1 mutants were assessed. It is thus possible that transgenes were overexpressed to levels that deliver nonphysiologic biologic responses, a concern expressed by the authors in the original publication. In the current report, Yan et al. (4) conducted an investigation analogous to the C. elegans experiment, expressing wild-type KSR1 or kinase-inactive KSR1 (Ki-KSR1 ${ }^{\mathrm{D} 683 / \mathrm{D} 700}$ ) in immortalized colon epithelial cell lines null for mouse KSR1 (KSR-/-MCE cells). At similar levels, wild-type KSR1 but not Ki-KSR1 1083/D700 restored TNF- $\alpha$ activation of MAPK, Akt/ $\mathrm{PKB}$, and IкB $\alpha$. Further, one possible explanation for the failure of Ki-KSR1 ${ }^{\text {D683/D70 to }}$ mimic wild-type KSR1, that substitution of the catalytic Asp with Ala rendered KSR1 incapable of binding MEK1 as occurred with Drosophila KSR, was ruled out in the current study and in another recently published report (25). These results provide strong support for the notion that the Ki-KSR1 ${ }^{\text {D683/D700 dominant-negative }}$ effect on c-Raf-1/MEK/MAPK activation in numerous mammalian systems is likely due to disruption of kinase activity. Further, these studies suggest that the mechanism of action of KSR orthologs may not be conserved across species. It is unlikely that C. elegans KSR-1 functions similarly to mammalian KSR1, as C. elegans KSR-1 appears to be a tyrosine kinase and the putative phosphorylation site on c-Raf-1 is not conserved in the C. elegans c-Raf-1 ortholog LIN-45 RAF.

\section{Concluding remarks}

Although the mechanism of the catalytic action of KSR1 kinase activity is undefined, recent studies confirm that it does bind ATP (44) despite substitution in subdomain II of Arg for the Lys that normally serves this function. Further, conversion of Arg to Lys, which should restore kinase activity to KSR1 if Arg in subdomain II were naturally inactivating, failed to alter the impact of KSR1 on germinal vesicle breakdown (12) or Elk-1 activation (45). While Lys is the amino acid most efficient in coordinating ATP within the ATP-binding pocket, as it has two hydrogen bonds to contact the $\alpha$ and $\beta$ phosphates of ATP, Arg and His can also serve as hydrogenbond donors (46). Further, recent studies showed that some kinases, including protein kinase C-ı (47), the Cak1-like cyclindependent kinase-activating kinases (48, 49), and WNK1 [for with no lysine (35)] (50-52), display full kinase activity irrespective of the conserved Lys in subdomain II, binding ATP by different strategies altogether. A detailed analysis of the manner by which ATP fits into the KSR1-binding pocket will be necessary to elucidate how KSR1 functions as a kinase.

The studies by Yan and colleagues provide strong evidence that KSR1 possesses biologically relevant kinase activity toward c-Raf- 1 that impacts the pathogenesis of IBD. While the scaffold model of KSR1 function is primarily based on the presumption of it not acting as a kinase (13, $15,17,19,41,42)$, this model should be reconsidered in view of the growing body of data supporting KSR1 kinase activity. A better understanding of the mechanism by which KSR1 signals may affect not only therapeutic approaches to IBD, but also to Ras-mediated tumorigenesis. In this regard, a KSR1 phosphorothioate antisense oligonucleotide is currently being developed by the National Cancer Institute (NCI) for the treatment of Kirsten Rasdriven human pancreatic cancer (Developmental Therapeutics Program/NCI Rapid Access to Intervention Development project BDP/486). Hopefully, the information provided by the current study will assist investigators interested in the mechanism by which KSR1 mediates Ras function to collaborate in addressing the issue of the kinase activity associated with KSR1.

\section{Acknowledgments}

This work was supported by grants CA42385 (to R. Kolesnick) and CA98022 (to H. Rosie Xing) from the NIH. We thank Zvi Fuks for critical reading of the manuscript.

Address correspondence to: Richard Kolesnick, Laboratory of Signal Transduction, Memorial Sloan-Kettering Cancer Center, 1275 York Avenue, New York, New York 10021, USA. Phone: (212) 639-7558; Fax: (212) 639-2767; E-mail: r-kolesnick@ski.mskcc.org.
1. Podolsky, D.K. 2002. Inflammatory bowel disease. N. Engl. J. Med. 347:417-429.

2. Kontoyiannis, D., Pasparakis, M., Pizarro, T.T., Cominelli, F., and Kollias, G. 1999. Impaired on/ off regulation of TNF biosynthesis in mice lacking TNF AU-rich elements: implications for joint and gut-associated immunopathologies. Immunity. 10:387-398.

3. Targan, S.R., et al. 1997. A short-term study of chimeric monoclonal antibody cA2 to tumor necrosis factor alpha for Crohn's disease. Crohn's Disease cA2 Study Group. N. Engl. J. Med. 337:1029-1035.

4. Yan, F., et al. 2004. Kinase suppressor of Ras-1 protects intestinal epithelium from cytokine-mediated apoptosis during inflammation. J. Clin. Invest. 114:1272-1280. doi:10.1172/JCI200421022.

5. Kaiser, G.C., Yan, F., and Polk, D.B. 1999. Conversion of TNF alpha from antiproliferative to proliferative ligand in mouse intestinal epithelial cells by regulating mitogen-activated protein kinase. Exp. Cell Res. 249:349-358.

6. Yan, F., John, S.K., and Polk, D.B. 2001. Kinase suppressor of Ras determines survival of intestinal epithelial cells exposed to tumor necrosis factor. Cancer Res. 61:8668-8675.

7. Yan, F., and Polk, D.B. 2001. Kinase suppressor of ras is necessary for tumor necrosis factor alpha activation of extracellular signal-regulated kinase/ mitogen-activated protein kinase in intestinal epithelial cells. Cancer Res. 61:963-969.

8. Zhang, Y., et al. 1997. Kinase suppressor of Ras is ceramide-activated protein kinase. Cell. 89:63-72.

9. Therrien, M., et al. 1995. KSR, a novel protein kinase required for RAS signal transduction. Cell. 83:879-888.

10. Kornfeld, K., Hom, D.B., and Horvitz, H.R. 1995. The ksr-1 gene encodes a novel protein kinase involved in Ras-mediated signaling in C. elegans. Cell. 83:903-913.

11. Sundaram, M., and Han, M. 1995. The C. elegans ksr-1 gene encodes a novel Raf-related kinase involved in Ras-mediated signal transduction. Cell. 83:889-901.

12. Therrien, M., Michaud, N.R., Rubin, G.M., and Morrison, D.K. 1996. KSR modulates signal propagation within the MAPK cascade. Genes Dev. 10:2684-2695.

13. Denouel-Galy, A., et al. 1998. Murine Ksr interacts with MEK and inhibits Ras-induced transformation. Curr. Biol. 8:46-55.

14. Xing, H., Kornfeld, K., and Muslin, A.J. 1997. The protein kinase KSR interacts with 14-3-3 protein and Raf. Curr. Biol. 7:294-300.

15. Yu, W., Fantl, W.J., Harrowe, G., and Williams, L.T. 1998. Regulation of the MAP kinase pathway by mammalian Ksr through direct interaction with MEK and ERK. Curr. Biol. 8:56-64.

16. Nguyen, A., et al. 2002. Kinase suppressor of Ras (KSR) is a scaffold which facilitates mitogen-activated protein kinase activation in vivo. Mol. Cell. Biol. 22:3035-3045.

17. Muller, J., Ory, S., Copeland, T., Piwnica-Worms, H., and Morrison, D.K. 2001. C-TAK1 regulates Ras signaling by phosphorylating the MAPK scaffold, KSR1. Mol. Cell. 8:983-993.

18. Ory, S., Zhou, M., Conrads, T.P., Veenstra, T.D., and Morrison, D.K. 2003. Protein phosphatase 2A positively regulates Ras signaling by dephosphorylating KSR1 and Raf-1 on critical 14-3-3 binding sites. Curr. Biol. 13:1356-1364.

19. Stewart, S., et al. 1999. Kinase suppressor of Ras forms a multiprotein signaling complex and modulates MEK localization. Mol. Cell. Biol. 19:5523-5534.

20. Bell, B., Xing, H., Yan, K., Gautam, N., and Muslin, A.J. 1999. KSR-1 binds to G-protein betagamma subunits and inhibits beta gamma-induced mitogen-activated protein kinase activation. J. Biol. Chem. 274:7982-7986 
21. Matheny, S.A., et al. 2004. Ras regulates assembly of mitogenic signalling complexes through the effector protein IMP. Nature. 427:256-260.

22. Wang, X., and Studzinski, G.P. 2001. Phosphorylation of raf- 1 by kinase suppressor of ras is inhibited by "MEK-specific" inhibitors PD 098059 and U0126 in differentiating HL60 cells. Exp. Cell Res. 268:294-300.

23. Xing, H.R., and Kolesnick, R. 2001. Kinase suppressor of Ras signals through Thr269 of c-Raf-1. J. Biol. Chem. 276:9733-9741.

24. Wang, X., and Studzinski, G.P. 2004. Kinase suppressor of RAS (KSR) amplifies the differentiation signal provided by low concentrations 1,25-dihydroxyvitamin D3. J. Cell. Physiol. 198:333-342.

25. Xing, H.R., Campodonico, L., and Kolesnick, R. 2004. The kinase activity of kinase suppressor of Ras1 (KSR1) is independent of bound MEK. J. Biol. Chem. 279:26210-26214.

26. Xing, H.R., Lozano, J., and Kolesnick, R. 2000 Epidermal growth factor treatment enhances the kinase activity of kinase suppressor of Ras. J. Biol. Chem. 275:17276-17280.

27. Galve-Roperh, I., et al. 2000. Anti-tumoral action of cannabinoids: involvement of sustained ceramide accumulation and extracellular signal-regulated kinase activation. Nat. Med. 6:313-319.

28. Grassme, H., Schwarz, H., and Gulbins, E. 2001. Molecular mechanisms of ceramide-mediated CD95 clustering. Biochem. Biophys. Res. Commun. 284:1016-1030.

29. Abdel Shakor, A.B., Kwiatkowska, K., and Sobota, A. 2004. Cell surface ceramide generation precedes and controls Fcgamma RII clustering and phosphorylation in rafts. J. Biol. Chem. 279:36778-36787.

30. van Blitterswijk, W.J. 1998. Hypothesis: ceramide conditionally activates atypical protein kinases C, Raf- 1 and KSR through binding to their cysteinerich domains. Biochem. J. 331:679-680.

31. Yao, B., et al. 1995. Phosphorylation of Raf by ceramide-activated protein kinase. Nature. 378:307-310.

32. Mathias, S., Dressler, K.A., and Kolesnick, R.N 1991. Characterization of a ceramide-activated protein kinase: stimulation by tumor necrosis factor alpha. Proc. Natl. Acad. Sci. U. S. A. 88:10009-10013.

33. Joseph, C.K., Byun, H.S., Bittman, R., and Kolesnick, R.N. 1993. Substrate recognition by ceramide-activated protein kinase. Evidence that kinase activity is proline-directed. J. Biol. Chem. 268:20002-20006.

34. Liu, J., Mathias, S., Yang, Z., and Kolesnick, R.N. 1994. Renaturation and tumor necrosis factoralpha stimulation of a $97-\mathrm{kDa}$ ceramide-activated protein kinase. J. Biol. Chem. 269:3047-3052.

35. Basu, S., Bayoumy, S., Zhang, Y., Lozano, J., and Kolesnick, R. 1998. BAD enables ceramide to signal apoptosis via Ras and Raf-1. J. Biol. Chem. 273:30419-30426.

36. Xing, H.R., et al. 2003. Pharmacologic inactivation of kinase suppressor of ras- 1 abrogates Ras-mediated pancreatic cancer. Nat. Med. 9:1266-1268.

37. Joneson, T., et al. 1998. Kinase suppressor of Ras inhibits the activation of extracellular ligandregulated (ERK) mitogen-activated protein (MAP) kinase by growth factors, activated Ras, and Ras effectors. J. Biol. Chem. 273:7743-7748.

38. Cacace, A.M., et al. 1999. Identification of constitutive and ras-inducible phosphorylation sites of KSR: implications for 14-3-3 binding, mitogenactivated protein kinase binding, and KSR overexpression. Mol. Cell. Biol. 19:229-240.

39. Kortum, R.L., and Lewis, R.E. 2004. The molecular scaffold KSR1 regulates the proliferative and oncogenic potential of cells. Mol. Cell. Biol. 24:4407-4416.

40. Lozano, J., et al. 2003. Deficiency of kinase suppressor of Ras1 prevents oncogenic ras signaling in mice. Cancer Res. 63:4232-4238.

41. Michaud, N.R. et al. 1997. KSR stimulates Raf-1 activity in a kinase-independent manner. Proc. Natl. Acad. Sci. U. S. A. 94:12792-12796.
42. Volle, D.J., et al. 1999. Phosphorylation of the kinase suppressor of ras by associated kinases. Biochemistry. 38:5130-5137.

43. Zhou, M., Horita, D.A., Waugh, D.S., Byrd, R.A., and Morrison, D.K. 2002. Solution structure and functional analysis of the cysteine-rich $\mathrm{C} 1$ domain of kinase suppressor of Ras (KSR). J. Mol. Biol. 315:435-446.

44. Muller, J., Cacace, A.M., Lyons, W.E., McGill, C.B., and Morrison, D.K. 2000. Identification of B-KSR1, a novel brain-specific isoform of KSR1 that functions in neuronal signaling. Mol. Cell. Biol. 20:5529-5539.

45. Sugimoto, T., Stewart, S., Han, M., and Guan, K.L. 1998. The kinase suppressor of Ras (KSR) modulates growth factor and Ras signaling by uncoupling Elk-1 phosphorylation from MAP kinase activation. EMBO J. 17:1717-1727.

46. Johnson, L.N., Lowe, E.D., Noble, M.E., and Owen, D.J. 1998. The Eleventh Datta Lecture. The structural basis for substrate recognition and control by protein kinases. FEBS Lett. 430:1-11.

47. Spitaler, M., Villunger, A., Grunicke, H., and Uberall, F. 2000. Unique structural and functional properties of the ATP-binding domain of atypical protein kinase C-iota. J. Biol. Chem. 275:33289-33296.

48. Tsakraklides, V., and Solomon, M.J. 2002. Comparison of Cak1p-like cyclin-dependent kinase-activating kinases. J. Biol. Chem. 277:33482-33489.

49. Kaldis, P., et al. 1998. Localization and regulation of the cdk-activating kinase (Cak1p) from budding yeast. J. Cell. Sci. 111:3585-3596.

50. Xu, B.E., et al. 2002. Regulation of WNK1 by an autoinhibitory domain and autophosphorylation. J. Biol. Chem. 277:48456-48462.

51. Xu, B., et al. 2000. WNK1, a novel mammalian serine/ threonine protein kinase lacking the catalytic lysine in subdomain II. J. Biol. Chem. 275:16795-16801.

52. Verissimo, F., and Jordan, P. 2001. WNK kinases, a novel protein kinase subfamily in multi-cellular organisms. Oncogene. 20:5562-5569.

\title{
p16 and ARF: activation of teenage proteins in old age
}

\author{
Ande Satyanarayana and K. Lenhard Rudolph
}

Department of Gastroenterology, Hepatology, and Endocrinology, Hannover Medical School, Hannover, Germany.

\begin{abstract}
Cellular senescence induced by different stresses and telomere shortening appears to play an important role in the aging process. The products of the INK4a/ARF locus - $\mathrm{p}^{16^{\mathrm{INK}} \mathrm{Ia}^{\mathrm{a}}}$ and ARF - arrest cell proliferation at the senescence stage by exerting their effects on retinoblastoma protein-and p53-mediated responsive pathways. A study in this issue of the JCI provides experimental evidence of a specific upregulation of these cell cycle inhibitors in a variety of organs during mammalian aging (see the related article beginning on page 1299).
\end{abstract}

Nonstandard abbreviations used: $C D K$, cyclin-dependent kinase; $\mathrm{CDKI}$, CDK inhibitor; $\mathrm{CR}$, caloric restriction; $\mathrm{GH}$, growth hormone; GHR, GH receptor; MDM2, mouse double minute 2 ; $\mathrm{pRB}$, retinoblastoma protein; SA- $\beta$-gal, senescence-associated $\beta$-galactosidase.

Conflict of interest: The authors have declared that no conflict of interest exists.

Citation for this article: J. Clin. Invest. 114:1237-1240 (2004). doi:10.1172/JCI200423437.
According to a current hypothesis on aging, senescent cells accumulate in the organism, and this results in failure of organ homeostasis and function (1). Cellular senescence - characterized by the permanent arrest of cell proliferation - is detrimental to the regenerative capacity of organs during aging. However, the senes- cence checkpoint is also considered to be a major mechanism for suppressing tumors, protecting the organism from cancer during early life (2). A number of stimuli have been identified as inducing senescence, and these include telomere shortening (3), DNA damage (4), oxidative stress (5), sustained mitogen stimulation (6), and other cellular stresses. Senescence induced by telomere shortening has been called "replicative senescence" and is a result of DNA damage-like signals generated by dysfunctional telomeres $(3,4)$. In addition to telomere attrition, several other mechanisms can abruptly induce senescence independent of telomere length, termed "premature senescence," including: over- 